\title{
Migrations, Mobilité et Territorialité chez les Tsiganes ${ }^{1}$
}

Alain Reyniers ${ }^{2}$

\begin{abstract}
$\mathrm{R}$ Lésumé: L'histoire des Tsiganes est liée à une série de mouvements migratoires, de déplacements d'immersion dans des territoires toujours occupés au préalable par des Gadjé. L'adaptation aux situations créés par la rencontre avec ces derniers a poussé les Tsiganes autant à la diversité qu'a l'établissement d'un rapport particuliers à espace fréquenté. Après avoir rappelé les grandes lignes de ce processus, l'auteur reprend chacune des trois notions de migration, de mobilité et de territorialité pour les caractériser et montrer leur complémentarité et leur richesse dans l'approche compréhensive du phénomène tsigane.

$\mathrm{R}$ Resumo: A história dos ciganos está associada a uma série de movimentos migratórios, de deslocaçōes e de implantaçōes em territórios sempre previamente ocupados pelos Gadjè. A adaptação às situações criadas pelo reencontro com estes, levou os ciganos a uma relação com o espaço particular e diversa. Após relembrar as grandes linhas deste processo, o autor retoma as noções de migração, mobilidade e territorialidade para as caracterizar e mostrar a sua complementaridade e a sua riqueza na abordagem compreensiva do fenómeno cigano.
\end{abstract}

Palavras-chave: Ciganos; Mobilidade; Nomadismo peripatético; Territorialidade.

Les Roms, les Manouches, les Sinti, les Gitans et les Voyageurs passent trop facilement pour un ensemble hétéroclite de nomades mal aimés, étrangers aux terres sur lesquelles ils sont amenés à se déplacer, des gens à l'économie douteuse dont la culture est réduite à quelques traits folkloriques. Les déplacements des uns passent pour du grand banditisme, la migration des autres pour une errance éternelle, une fuite éperdue, la quête d'un Eldorado hypothétique.

\section{Histoire et diversité des formes d'implantations tsiganes}

Pour contrer ces stéréotypes, il convient de rappeler quelques certitudes d'ordre historique et ethnographique. Les Tsiganes sont originaires du nord-ouest de l'Inde. Lorsqu'ils apparurent en Europe orientale (alors Empire byzantin), sans doute dans le courant du xilème ou du xiIIème siècle, ils n'affichaient probablement pas tous un mode de vie itinérant. Néanmoins, ceux qui déambulaient étaient apparemment nombreux et c'est en tant que nomades que leurs descendants seront un peu partout, plus tard, distingués des autres populations. Néanmoins, leur liberté de mouvement connaîtra, assez vite, des contraintes: la réduction à l'esclavage dans les principautés roumaines de Moldavie et de Valachie, le rejet des collectivités locales en Europe centrale et occidentale, l'élaboration de politiques assimilationnistes menées contre eux en Espagne d'abord (dès 1499), dans l'Empire des Habsbourgs ensuite (seconde moitié du XvIIIème siècle).

Certains groupes tsiganes vont donc maintenir un mode de vie nomade, axé sur la fourniture de prestations temporaires aux Gadjé (les non-Tsiganes) et l'entretien d'une vie collective forte. Les autres vont davantage perdre leur mobilité, parfois aussi leur autonomie, et ils se retrouveront souvent au bas de l'échelle sociale comme petites mains résignées des seigneurs, des paysans et des citadins. Certains vont poursuivre un cheminement séparé en maîtrisant, autant que faire se peut, les

\footnotetext{
Este artigo com o mesmo título foi publicado em Bernard Drobenko (dir.), Territoires et minorités: la situation des Gens du Voyage. Actes du colloque des 25-26 mars 2004. Limoges, 2005, Presses de l'Université de Limoges, pp. 63-71.

2 Professeur à l'Université Catholique de Louvain; Directeur de la revue Études Tsiganes.
} 
moments de contact avec les populations avoisinantes. D'autres vont se replier sur eux-mêmes en vivotant au coup par coup à la périphérie des sociétés établies. Dans ce processus, quelques traits collectifs comme la propension à se garder du monde extérieur, la tendance à choisir les conjoints dans le seul milieu d'origine, le fatalisme face aux évènements, vont parfois être accentués et, en tout cas, vont jouer sur la séparation avec les autres collectivités. L'éducation des enfants va se maintenir dans la méfiance des Gadjé, toute tournée vers l'imitation des parents et l'exploration pratique de l'environnement.

De cette histoire en Europe, les Tsiganes auront hérité une diversité de situations, des formes différentes d'insertion territoriale et sociale. Aujourd'hui, le nomadisme subsiste comme tel dans différents pays d'Europe occidentale (en France, en Belgique, en Italie, en Angleterre ou en Irlande), moins bien en Europe centrale et orientale (à l'exception de la Roumanie où vivent encore un certain nombre de familles foncièrement nomades). La sédentarisation ne s'est pas nécessairement soldée par une perte de la mobilité économique (avec les moyens technologiques contemporains, il est possible de se déplacer plus vite et plus loin que par le passé, s'engager dans des opérations commerciales sans déplacer toute la famille). Pour autant, la plupart des Tsiganes se sont bel et bien installés, les uns dans des conditions qui ne les distinguent guère des Gadjé, les autres à la marge, parfois à la limite du supportable. Pour beaucoup, la qualité du niveau de vie s'est fortement dégradée au cours de ces dernières années et il en a résulté un isolement social croissant, le tout dans un contexte de xénophobie et de perte de repères identitaires. Aucun pays d'Europe n'échappe à ce phénomène. Mais c'est en Europe de l'Est, là où les Tsiganes sont les plus nombreux, qu'il est le plus flagrant.

\section{Migrations}

Les migrations poussent des populations d'une terre à une autre, sans véritable espoir de retour. Il y a un départ suivi d'une transplantation dans un autre univers. Chez les Tsiganes, comme pour la plupart des êtres humains, ce phénomène est assez rare et plutôt soumis à des circonstances politiques, économiques ou sociales majeures. Le départ de l'Inde vers l'Empire byzantin, puis l'essaimage dans les Balkans d'abord (seconde moitié du XIVème siècle), en Europe centrale ensuite (début du Xvème siècle), puis dans l'Europe entière (fin du Xvème siècle) a défini une première migration des Tsiganes. Une seconde migration importante parce que planétaire - est celle qui a poussé des Roms sur toutes les routes du monde, dès la suppression de l'esclavage dans les principautés roumaines, au milieu du XIXème siècle. En 1866, profitant de la guerre entre la Prusse et l'Autriche-Hongrie, certains parmi ces Roms suivent les armées, pénètrent en Allemagne, puis en Belgique et en France. Leurs habitudes assez différentes de celles des nomades habitués à l'Occident vont être invoquées par les pouvoirs publics pour justifier une lutte contre les vagabonds, les nomades et autres populations mobiles qualifiées de dangereuses. Après la Seconde Guerre Mondiale, au cours de laquelle les Tsiganes connaîtront un génocide analogue à celui des Juifs, une troisième migration, peu voyante, va se développer au départ de la Yougoslavie. Profitant de l'assouplissement des frontières de l'Etat yougoslave au début des années soixante, plusieurs dizaine de milliers de Tsiganes originaires de Serbie, de Bosnie ou de Macédoine vont s'installer dans les pays occidentaux comme travailleurs immigrés sédentarisés. Ils seront progressivement suivi, au cours de la décennie suivante, par d'autres Tsiganes yougoslaves beaucoup plus mobiles.

Un quatrième mouvement migratoire de Tsiganes, démarre à la fin des années quatre-vingt avec l'arrivée de Roms, pour la plupart originaires de Macédoine, en Allemagne. Placé sous la bannière des Droits de l'Homme, il va annoncer la venue actuelle de tous ces Roms demandeurs d'asile polonais, bulgares, slovaques et surtout roumains. Ce mouvement est lié à la chute des régimes communistes en Europe centrale et orientale. D'abord perçus comme sous-prolétaires issus d'une classe pauvre, caractérisée par un mode de vie primitif, les Tsiganes auront connu des sorts divers dans les Etats socialistes. Certains pourront gravir les échelons de la vie sociale, d'autres réussiront à conserver une liberté de mouvements. Mais le nomadisme aura été combattu et interdit un peu partout. Transformés en main d'oeuvre peu qualifiée, la plupart des Tsiganes seront fixés et occupés à des tâches subalternes, dangereuses 
ou insalubres, peu valorisantes. Aujourd'hui, alors même que l'Europe orientale s'engage dans une transition vers le libéralisme, ils sont massivement licenciés et souvent victimes de frustrations xénophobes ou d'un rejet ultranationaliste. Dans ce contexte, l'Ouest peut très bien leur apparaître comme un nouvel Eldorado qui stimule de nouvelles perspectives migratoires.

Les mouvements migratoires qui touchent les Tsiganes à l'époque contemporaine ne traduisent donc pas du tout la reprise fantasmagorique d'une errance collective. Les uns sont liés à la recherche de débouchés économiques nouveaux, les autres dépendent plutôt d'une volonté de fuir des situations politiques, ou sociales parfois insoutenables. Souvent, les mouvements migratoires sont menés sous l'impulsion de causes diverses. Des familles qui se déplacent vers l'ouest de l'Europe en quête de ressources peuvent très bien trouver dans les atteintes aux droits de l'Homme qui touchent certains de leurs proches un argument supplémentaire pour accélérer le départ. De même, les perspectives de gains substantiels en Occident ne sont pas nécessairement plus motivants que l'opportunité de se créer un capital de contacts, de considération et de ressources culturelles.

\section{Nomadisme et mobilité}

Bien qu'il puisse, dans ses effets, favoriser un déplacement progressif de population d'un territoire à l'autre, le nomadisme est d'une toute autre nature que la migration. Il constitue, en effet, une forme particulière d'exploitation des ressources disponibles sur un territoire et il s'appuie sur la circulation des individus au sein d'entités sociales qui organisent l'accès à cette exploitation. Etant donné la liste des activités traditionnelles exercées par les Tsiganes et attestées parfois depuis leur arrivée en Europe (chaudronniers, ferronniers, vanniers, maquignons, devins, artistes, musiciens, commerçants ambulants...) la plupart de leurs déplacements relève du nomadisme dit péripatétique. Il ne s'agit pas d'exploiter directement les ressources offertes par la Nature, mais bien de capter, par un jeu d'échanges, celles qui résultent du travail des populations déjà installées sur un territoire donné. Le nomadisme péripatétique se distingue par la fourniture intermittente de marchandises, de services et de main d'œuvre à une clientèle dispersée dont les besoins à satisfaire sont occasionnels. Le profil économique du Tsigane est celui du travailleur indépendant, maître de son temps de travail, de sa force de travail et de ses mouvements, qui déniche les besoins ou les suscite. Bien qu'il puisse se spécialiser dans un domaine où il excelle (la forge, les arts du cirque...), le Tsigane est le plus souvent tenu à une polyvalence élémentaire, afin de saisir les affaires qui se présentent à lui (il sera tour à tour marchand de tapis, brocanteur, intermédiaire dans une vente de voiture...) ou de s'adapter aux saisons (cueillette des agrumes, vendanges...).

Liée à la nécessité de se déplacer, la polyvalence pousse au choix d'activités qui ne nécessitent pas un outillage volumineux et sophistiqué, qui porte sur le négoce de marchandises aisément transportables, qui demandent un travail intensif, de courte durée, dont le rendement est immédiat. Le plus souvent, la réussite économique des Tsiganes est subordonnée à la capacité de cerner les affaires opportunes et à la maîtrise d'un jeu relationnel. Le Tsigane doit prendre l'initiative du contact économique avec les Gadjé (les non-Tsiganes). Il doit être à même de jouer un rôle, persuader, «baratiner», faire preuve d'audace et de persévérance. Certaines activités, très voyantes, comme la mendicité ou le ramassage de la ferraille, ne nécessitent pas toutes ces qualités. Bien qu'elles fassent parfois aussi l'objet d'une véritable spécialisation, elles sont souvent temporaires, liées à la recherche d'une autre forme d'insertion économique ou faute de mieux.

Un Tsigane vit rarement seul. Là où il ne peut faire face à une demande, là où ses capacités individuelles font défaut, il trouve toujours quelqu'un de son entourage pour le seconder. Le Tsigane voyage avec sa famille (son épouse, ses enfants et beaux enfants, mais aussi ses frères, ses cousins, des parents plus éloignés). Le noyau familial est fréquemment associé, de manière temporaire, à d'autres noyaux qui forment des ensembles sociaux plus vastes et mouvants, au gré des va-et-vient. C'est au sein de ces ensembles que chaque Tsigane trouve des possibilités d'entraide et de solidarité, au besoin en jouant sur ses relations de parenté. L'édifice social qui résulte du mouvement des familles est parcouru de tensions multiples: la collectivité, qui pousse à l'unanimité de ses membres, notamment à l'harmonisation du niveau de 
vie, à l'adoption de valeurs similaires, à sa pérennité dans le resserrement des liens matrimoniaux n'est pas toujours soutenue par les cellules familiales qui ont sur tous ces points leurs exigences propres. Les périodes de co-circulation succèdent aux périodes de co-résidence. Lorsque la clientèle est suffisamment concentrée, mais aussi sous la pression des Gadjé, parfois à la suite d'un progrès technique ou d'un changement économique majeur, les collectivités se sédentarisent. Les politiques assimilationnistes menées un peu partout à l'égard des Tsiganes ont, de tout temps, recherché ce but. L'adoption de la traction automobile a récemment favorisé la stabilisation: en effet, il est devenu possible de se déplacer plus vite et plus loin qu'à l'époque de la traction hippomobile et de revenir le soir là où la famille est restée. La nécessité du stockage des marchandises a, elle aussi, poussé à l'acquisition d'un lopin de terre ou d'une maison. Mais, quoi qu'il en soit, la circulation demeure. Parce que les activités économiques ont peu changé et nécessitent toujours un déplacement vers la clientèle. Mais aussi parce que toutes les cellules familiales cherchent à maintenir des contacts avec des parents et des amis installés ailleurs.

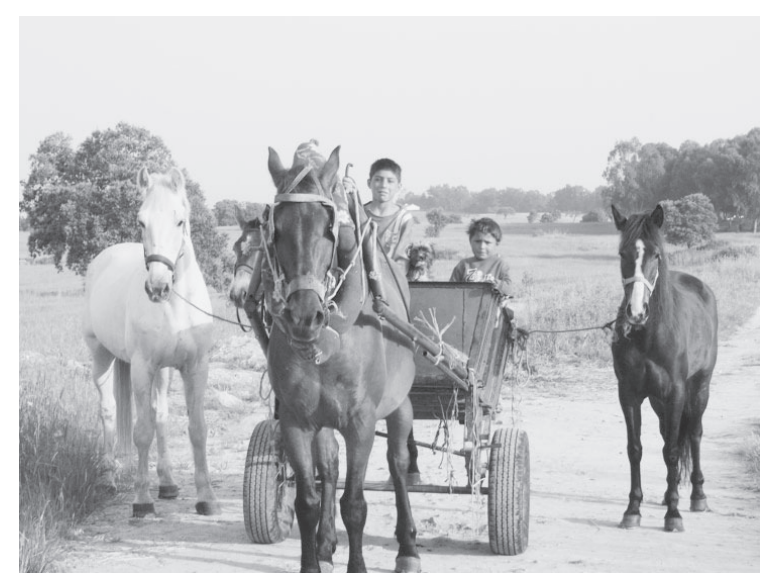

Fonte: André Correia

La mobilité tsigane propose dès lors des contours très variables: itinérance collective dans la longue durée, stabilité résidentielle mais circulation à caractère familial et économique, déplacement résidentiel mais fourniture d'une clientèle attitrée, stabilité résidentielle et économique mais visites incessantes des parents... Dans certains cas, tel celui des agriculteurs tsiganes d'Europe centrale, la circulation s'est apaisée mais l'endogamie contribue au maintien d'une identité collective et singulière. Organisés en familles qui articulent leurs mouvements au sein de communautés plus ou moins stables et qui, dans ce contexte, développent par un système d'entraide l'accès aux ressources disponibles, les Tsiganes n’ont rien de groupes errants. Bien insérés dans un réseau complexe d'alliances, ils développent aussi une insertion territoriale effective. Autrement dit, la densité du maillage de leurs liens sociaux s'accompagne d'un enracinement dans un territoire particulier.

\section{Territorialité}

Ce territoire est d'abord celui de Gadjé, les clients mais aussi ceux qui rejètent. C'est dans les interstices laissées libres par les Gadjé qu'apparaît le territoire tsigane. Suivant le mode de vie, les habitudes, il est parsemé de pôles d'attraction, familiaux (là où habitent les parents, mais aussi les cimetières où sont enterrés les parents disparus), religieux, administratifs (préfectures, hôpitaux...) et bien sûr économiques (grossistes, marchés...). Les déplacements se font alors d'un pôle à l'autre avec, à la limite, toute la routine prêtée à la stabilité. Mais, parfois, ces pôles ne sont qu'occasionnels, n'impliquent pas ou peu de familiarité. Ils s'insèrent dans une suite de points de rencontre, de lieux de halte occasionnels, d'endroits où l'on développe toute une série d'activités temporaires. Le territoire prend l'allure d'un parcours toujours en formation, toujours renégocié au sein des communautés et avec les Gadjé (ou leurs autorités). L'insertion dans un territoire de polarisation ou dans un territoire de parcours est autant liée à des formes particulières d'exploitation des ressources (la mendicité implique moins de connivence avec une population que les activités qui nécessitent plus de stratégie commerciale), qu'à l'extension du réseau familial et à des prédispositions psychologiques. Aussi ne peut-on distinguer ces territoires à l'étendue de l'espace qu'ils occupent. Un territoire de parcours peut se limiter à deux ou trois cantons comme il peut s'étendre à l'Europe entière. Un territoire de polarisation peut être limité à quelques grosses bourgades contiguës comme à des villes distantes de plusieurs centaines de kilomètres. L'étroite imbrication des uns et des autres (on peut, dans une même ville, rencontrer des Manouches ou des Gitans polarisés et des Roms roumains ou d'autres Manouches pérégrinant) est pour beaucoup dans la difficulté de cerner l'univers tsigane. 
Le mouvement pousse à la dispersion autant qu'à la concentration. Il est parfois impulsé sur plainte des Gadjé qui ne peuvent tolérer la présence des tsiganes. Les contrôles précèdent alors la traque et les expulsions. Mais le rapport au territoire est bien plus encore lié aux rapports de parenté entre les Tsiganes qui le fréquentent. Des relations durables existent entre des frères, des cousins, des amis et se prolongent par des alliances entre certains de leurs enfants. On assiste alors fréquemment à des «renchaînements d'alliance» tout à fait caractéristiques: des frères et des sœurs d'une famille prennent les sœurs et les frères d'une autre famille. Il y a même de véritables unions triangulaires lorsque les enfants d'une famille choisissent leurs conjoints dans des familles que rapprochent déjà d'autres alliances. Il y a là tout un processus qui joue sur plusieurs générations. Une tendance nette à l'endogamie apparaît. En même temps, les choix personnels, le trop grand nombre d'enfants (il y a parfois un écart d'une vingtaine d'années entre l'aîné et le benjamin), les circonstances de l'existence (un divorce, un changement de vie) peuvent pousser à l'éclatement, à l'exogamie. Néanmoins, les risques de dilution massive parmi les Gadjé sont minces et la conscience d'appartenir à une société qui transcende le temps demeure forte. Pour peu que la mémoire de liens antérieurs entre individus qui se retrouvent fortuitement ait été entretenue, un cheminement collectif reprendra tôt ou tard.

Ce processus de dispersion et de regroupement, perceptible dans la longue durée, est également apparent dans le court terme. Tous les membres de la communauté maintiennent des contacts de densité variable. L'observation des lieux de halte et de la composition des convois montre une intense circulation d'individus dont les itinéraires se croisent et se recroisent, qui butinent d'un regroupement temporaire à l'autre, qui séjournent à proximité d'un parent sédentarisé ou qui ne négligent pas de le visiter. Le territoire se présente bien comme un ensemble de circuits qui dessinent un maillage très serré de l'espace. La volonté de ne pas vivre isolé de ses proches et de les entourer en cas de besoin contribue puissamment à la recherche de moments d'intense fusion. Mais, à l'inverse, le désir de partir, pour échapper à un conflit, aller voir d'autres proches installés ailleurs, découvrir de nouvelles choses ou tout simplement pour bouger, est également très fort. Le groupe respire au gré de ces va-et-vient.
Trop souvent, l'espace tangible des Tsiganes est mis à la marge des société «installées»: sur les dépotoirs, sur les terrains vagues et sur les portions de routes abandonnées à l'Ouest; à la sortie des villages ou dans des quartiers d'immeubles délabrés à l'Est, stigmates d'une existence marginale pour les Gadjé. L'espace tsigane n'est pas pour autant réductible à cet aspect des choses. C'est aussi, et surtout, un lieu de vie, ouvert sur des pratiques sociales, économiques, relationnelles, maîtrisées par ceux qui les déploient. Ce lieu, c'est l'endroit où les membres d'une communauté ont leurs habitudes, là où ils ont tendance à se retrouver. Tantôt, il s'agit de l'endroit où s'est sédentarisé un membre de la famille autour duquel vont et viennent tous les autres parents. Tantôt, il s'agit d'un terrain de stationnement, d'une maison ou d'un lopin de terre qui a été acheté et plus ou moins aménagé. Certains de ces endroits prennent plus d'importance que d'autres, car s'y accumulent aux alentours des possibilités de travail, des services administratifs et hospitaliers, une infrastructure scolaire appréciée. D’autres sont tout à coup abandonnés, parce qu'un conflit est survenu, parce qu'un décès y a eu lieu. Tous ces endroits se révèlent riches en significations, le plus souvent totalement méconnues des Gadjé Partout où ils s'installent, les Tsiganes ont, en effet, tendance à reproduire dans l'espace, les éléments qui structurent leur rapport au territoire collectif: la disposition longitudinale ou alvéolaire des caravanes qui évoque le passage ou l'installation plus durable; le choix des emplacements qui tient compte autant du relief que des rapports de proximité ou d'évitement avec les voisins; le déploiement de la vie domestique et collective.

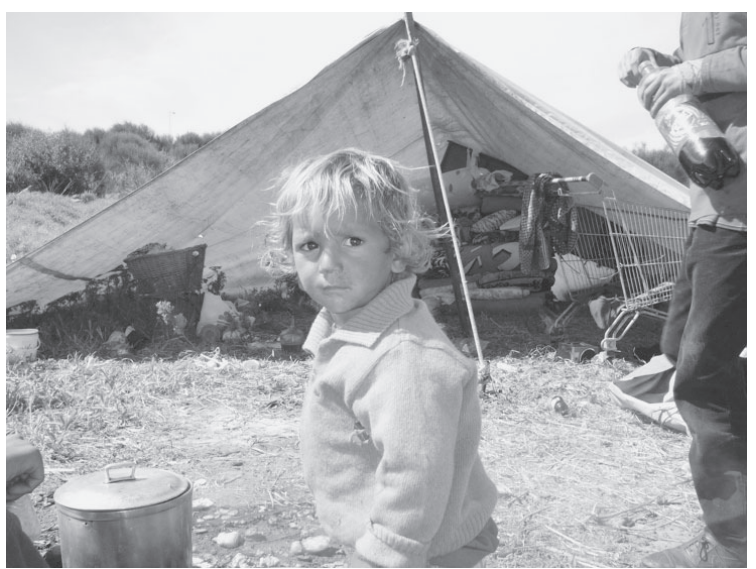

Fonte: André Correia 


\section{Orientations bibliographiques}

ASSEO, (H.), 1994: Les Tsiganes, une destinée européenne. Gallimard, coll. Découvertes, Paris.

COMITÉ EUROPÉEN SUR LES MIGRATIONS (CDMG), Problèmes liés à la mobilité internationale des Roms en Europe. 1. Rapport préparé par Dr. Yaron Matras, Université de Manchester (manuscrit soumis le 15 décembre 1996). 2. Conclusions adoptées par le Comité européen sur les migrations (CDMG) lors de sa 36ème réunion (avril 1997), Conseil de l'Europe, Strasbourg, 1998.

FRASER (A.), The Gypsies, Blackwell, Oxford (UK) et Cambridge (UK), 1992.

GHEORGHE (N.) et MIRGA (A.), The Roma in the Twenty-First Century: a Policy Paper, Project on Ethnic Relations, Princeton (USA), 1997.

HUMEAU, (J.-B.), 1995: Tsiganes en France, de l'assignation au droit d'habiter. L'Harmattan, Paris.

KENRICK, (D.), PUXON, (G.), 1974: Destins gitans, des origines à la solution finale. Calmann-Lévy, Paris.

LIEGEOIS, (J.-P.), 1994: Roma, Tsiganes, Voyageurs. Conseil de l'Europe, Strasbourg.
MARCHAND, (A.), 2001: La protection des droits des Tsiganes dans l'Europe d'aujourd'hui. L'Harmattan, Paris.

MATRAS (Y.), "Romani Migrations in the Post-Communist Era: their Historical and Political Signifiance", Cambridge Review of International Affairs, Vol. XIII, n. ${ }^{\circ} 2,2000$, pp. 32-50.

REYNIERS (A.), 1995: Les populations tsiganes et leurs mouvements dans les pays d'Europe centrale et orientale et vers quelques pays de l'OCDE, OCDE (Migration Internationale et Politique du Marché du Travail, Document hors-série, n. ${ }^{\circ}$, Diffusion générale), Paris.

REYNIERS, (A.), 1998: Tsigane, heureux si tu es libre! UNESCO, coll. Mémoire des Peuples, Paris.

REYNIERS, (A.), 1998: "Le souçi de soi ou la perennité d'une communauté tsigane", Etudes Tsiganes, nouvelle série, VI, nr. 1 (L'urbanité réconciliée. L'habitat adapté: un enjeu de citoyenneté): 116-124.

REYNIERS, (A), WILLIAMS, (P.), 2001: "Permanence tsigane et politique de sédentarisation dans la France de l'après-guerre", in Etudes Tsiganes, vol. 15, (Les virtualités de la loi Besson, l'habitat saisi par le droit), p. 10-25. 